\title{
A FIBER HOMOTOPY EXTENSION THEOREM
}

\author{
BY \\ GUY ALLAUD AND EDWARD FADELL( $\left.{ }^{1}\right)$
}

1. Introduction. Our objective in this paper is to set forth a simple but very useful fiber homotopy extension theorem in the category of fiber spaces in the sense of Hurewicz [5]. We will give several applications of the theorem as well as point out that a related theorem used by A. T. Lundell in [7] is false. Fortunately, the theorem as we give it is strong enough to imply the validity of Lundell's techniques. Throughout this paper all fiber spaces $\mathcal{F}=(E, p, B)$ will denote Hurewicz fiber spaces with total space $E$, base space $B$, and fiber $\operatorname{map} p: E \rightarrow B . F_{b}=p^{-1}(b)$ will denote the fiber over $b \in B$. $B$ will will also be assumed 0 -connected so that all fibers are of the same homotopy type [1].

Finally, all fiber spaces and lifting functions [5] will be assumed to be regular, i.e., if $\Omega_{p}=\{(e, \omega) \mid p(e)=\omega(0)\} \subset E \times B^{I}$, then the lifting functions $\Lambda: \Omega_{p} \rightarrow E^{I}$ will have the additional property that $\Lambda(e, p(e))=e$, where $p(e)$ is identified with the constant path at the point $p(e) \in B$. Regularity is no restriction if $B$ is metric or if $\mathcal{F}$ is locally trivial and $B$ is paracompact [5].

\section{A fiber homotopy extension theorem.}

Theorem (2.1) (FHET). Let $\mathcal{F}=(E, p, B)$ be a fiber space and let $(X, A)$ be a metric pair ( $A$ closed in $X)$. Suppose there exists a map $G:(X \times\{0\})$ $\cup(A \times I) \rightarrow E$ such that $p G(x, t)=p G(x, 0), x \in A, 0 \leqq t \leqq 1$. Then if either $E$ is an $\mathrm{ANR}\left({ }^{2}\right)$ or $(X, A)$ is an ANR pair, $G$ can be extended to a map $H: X \times I \rightarrow E$ such that $p H(x, t)=p H(x, 0), x \in X, 0 \leqq t \leqq 1$.

Proof. Using a suitable homotopy extension theorem $G$ can be extended to a map $G^{\prime}: X \times I \rightarrow E$. $G^{\prime}$ can be regarded as a map $X \rightarrow E^{I}$. For $\alpha \in E^{I}$, $t \in I$, let $\alpha_{t} \in E^{I}$ be the path given by

$$
\alpha_{t}(s)=\alpha((1-s) t), \quad 0 \leqq s \leqq 1 .
$$

Then, $\alpha_{0}(s)=\alpha(0), \alpha_{1}(s)=\alpha(1-s), \alpha_{t}(0)=\alpha(t), \alpha_{t}(1)=\alpha(0)$. Let $\Lambda$ be a lifting function for $\mathcal{F}$ and define a homotopy $H: X \times I \rightarrow E$ by

$$
H(x, t)=\Lambda\left(G^{\prime}(x)(t), p\left(G^{\prime}(x)\right)_{t}\right)(1) .
$$

$H(x, 0)=\Lambda\left(G^{\prime}(x)(0), p\left(G^{\prime}(x)\right)_{0}\right)(1)=G^{\prime}(x)(0)=G(x, 0)$, since $\Lambda$ is regular. If $x \in A$, then $p\left(\left(G^{\prime}(x)\right)_{t}\right)(s)=p G(x, 0)$ and again by regularity $H(x, t)=G(x, t)$.

Received by the editors September 11, 1961.

(1) This work was supported in part by the National Science Foundation under Grant NSF G-11297.

(2) We consider here only separable metric ANR's. 
Finally, $p H(x, t)=p\left(\left(G^{\prime}(x)\right)_{t}\right)(1)=p G(x, 0)=p H(x, 0)$, and $H$ is the required map.

Let $\mathcal{F}=(E, p, B)$ be a fiber space and let $X$ be an arbitrary topological space. Two maps $h, g: X \rightarrow E$ such that $p g=p h$ are said to be fiber homotopic $(h \sim f g)$ if there is a map $H: X \times I \rightarrow E$ such that $H(x, 0)=h(x), H(x, 1)=g(x)$ and $p H(x, t)=h(x)=g(x), 0 \leqq t \leqq 1$.

In terms of the above notation the FHET can be restated as follows:

(2.2) FHET. Let $\mathcal{F}=(E, p, B)$ be a fiber space and let $(X, A)$ be a metric pair. Suppose that either $E$ is an ANR or $(X, A)$ is an ANR pair. Furthermore, suppose $h \sim_{f} g: A \rightarrow E$ and $h$ is extendable to a map $h^{\prime}: X \rightarrow E$. Then $g$ is extendable to a map $g^{\prime}: X \rightarrow E$ such that $h^{\prime} \sim_{f} g^{\prime}$.

The theorem should be compared with Theorem 2.1 of [2].

In [7, Theorem 2.1] A. T. Lundell states the following theorem and indicates that the proof is a slight modification of the proof of a suitable covering homotopy theorem.

Theorem (2.3). Let $\mathcal{F}=(E, p, B)$ be a locally trivial fiber space, let $X$ be locally compact and paracompact, and let $A$ be a closed subset of $X$. Suppose $f: X \rightarrow E$ and $h: X \times I \rightarrow B$ are such that $p f=h \mid X \times\{0\}$. Suppose further that there is a "partial lifting" of $h$ on $A$, i.e., a map $h^{\prime}: A \times I \rightarrow E$ such that $p h^{\prime}$ $=h \mid A \times I$, and $h^{\prime}|A \times\{0\}=f| A$. Then there exists a map $h: X \times I \rightarrow E$ such that $p \tilde{h}=h, \tilde{h} \mid X \times\{0\}=f$ and $\tilde{h} \mid A \times I=h^{\prime}$.

It is easy to see that such a theorem implies that all spaces have the homotopy extension property with respect to locally compact, paracompact pairs $(X, A)$ as follows. Let $E$ denote any topological space, $B$ a single point and $p: E \rightarrow B$ the constant map. $(E, p, B)$ is clearly a locally trivial fiber space. Now if $h^{\prime}:(X \times\{0\}) \cup(A \times I) \rightarrow E$ is given, $p h^{\prime}$ is clearly extendable to $h: X \times I \rightarrow B$. Applying the above theorem, $h^{\prime}$ is extendable to $\tilde{h}: X \times I \rightarrow E$. Hence, $E$ has the homotopy extension property with respect to $(X, A)$. This is clearly false since there are numerous examples of spaces which do not have the homotopy extension property with respect to compact pairs. We see here also that conditioning the base $B$ in the above theorem is of no consequence. We can, however, prove the following theorem which follows easily from FHET and which is sufficient for the material in [7].

TheOREM (2.4). Let $\mathcal{F}=(E, p, B)$ be a fiber space and $(X, A)$ a metric pair. Suppose that $E$ is an ANR or that $(X, A)$ is an ANR pair. Suppose further that there are maps $f:(X \times\{0\}) \cup(A \times I) \rightarrow E$ and $h: X \times I \rightarrow B$ such that $p f=h \mid(X \times\{0\}) \cup(A \times I)$. Then, there exists a map $\tilde{f}: X \times I \rightarrow E$ which is an extension of $f$ and such that $p \bar{f}=h$.

Proof. Let $h_{x} \in B^{I}$ and $f_{x} \in E^{I}$ be the paths defined by

$$
\begin{array}{ll}
h_{x}(t)=h(x, t), & x \in X, 0 \leqq t \leqq 1, \\
f_{x}(t)=f(x, t), & x \in A, 0 \leqq t \leqq 1 .
\end{array}
$$


Let $\Lambda$ be a lifting function for $\mathcal{F}$ and define a map $h^{\prime}: X \times I \rightarrow E$ by

$$
h^{\prime}(x, t)=\Lambda\left(f(x, 0), h_{x}\right)(t) .
$$

Then, $h^{\prime}(x, 0)=f(x, 0)$, and if $x \in A, h^{\prime}(x, t)=\Lambda\left(f_{x}(0), p f_{x}\right)(t)$ so that by Proposition 1, [1], there is a homotopy $H:(A \times I) \times I \rightarrow E$ with the following properties:

$$
\begin{aligned}
H(x, t, 0) & =h^{\prime}(x, t), \\
H(x, t, 1) & =f(x, t), \\
H(x, 0, s) & =h^{\prime}(x, 0)=f(x, 0), \\
p H(x, t, s) & =p H(x, t) .
\end{aligned}
$$

Define a map $H^{\prime}:(A \times I) \times I \cup(X \times\{0\}) \times I \rightarrow E$ by

$$
\begin{array}{ll}
H^{\prime}(x, t, s)=H(x, t, s), & x \in A, 0 \leqq t, s \leqq 1, \\
H^{\prime}(x, 0, s)=f(x, 0), & x \in A, 0 \leqq s \leqq 1 .
\end{array}
$$

Applying the FHET using the pair $(X \times I,(A \times I) \cup(X \times\{0\}))$, we obtain a map $\tilde{f}: X \times I \rightarrow E$, which is an extension of $f$, and which satisfies the relation $p \tilde{f}(x, t)=p h^{\prime}(x, t)=h(x, t)$. This proves the theorem.

Let us recall also the definition of a locally trivial fiber subspace. Let $\mathcal{F}=(E, p, B, F)$ and $\mathcal{F}^{\prime}=\left(E^{\prime}, p^{\prime}, B, F^{\prime}\right)$ be locally trivial fiber spaces over the same base $B . \mathcal{F}^{\prime}$ is called a locally trivial fiber subspace of $\mathcal{F}$ provided $E^{\prime} \subset E$, $p^{\prime}=p \mid E$ and for each $x \in B$ there is an open set $U$ and a homeomorphism of pairs $\phi_{U}:\left(U \times F, U \times F^{\prime}\right) \rightarrow\left(p^{-1}(U), p^{\prime-1}(U)\right)$ such that $p \phi_{U}(b, z)=b,(b, z)$ $\in U \times F$.

Example. Let $\mathcal{F}=(E, p, B, F)$ denote a locally trivial fiber space with fiber $F$ a manifold. Suppose $F$ admits a cross section $\sigma$ and $E^{\prime}=E-\sigma(B)$. Then, if $p^{\prime}=p \mid E^{\prime}, \mathcal{F}^{\prime}=\left(E^{\prime}, p^{\prime}, B, F^{\prime}\right)$ is a locally trivial fiber subspace of $\mathcal{F}$, where $F^{\prime}$ is $F$ minus a point.

An immediate consequence of the proof of the Uniformization Theorem of Hurewicz [5] gives the following.

Theorem (2.5). If $\mathfrak{F}^{\prime}=\left(E^{\prime}, p^{\prime}, B, F^{\prime}\right)$ is a locally trivial subspace of $\mathcal{F}=(E, p, B, F)$, then if $B$ is paracompact $\mathcal{F}$ possesses a (regular) lifting function $\Lambda$ with the additional property that $\Lambda\left(e^{\prime}, \omega\right) \subset E^{\prime \prime}$, whenever $e^{\prime} \in E^{\prime}$, i.e., $\Lambda$ restricted to $\mathfrak{F}^{\prime}$ serves as a (regular) lifting function for $\mathcal{F}^{\prime}$.

The following corollary is an immediate consequence of Theorem (2.5). It should be compared with Corollary 2.2 of Lundell [7] which was "proven" using the false Theorem (2.3).

Corollary (2.6). Let $\mathcal{F}^{\prime}=\left(E^{\prime}, p^{\prime}, B, F^{\prime}\right)$ denote a locally trivial fiber subspace of $\mathcal{F}=(E, p, B, F)$ with $B$ paracompact. Let $(X, A)$ denote a pair of topological spaces $(A$ need not be closed in $X), f:(X, A) \rightarrow\left(E, E^{\prime}\right)$ and $h: X \times I \rightarrow B$ maps 
such that $p f=h \mid X \times\{0\}$. Then, there exists a map $\tilde{h}:(X \times I, A \times I) \rightarrow\left(E, E^{\prime}\right)$ such that $\tilde{h} \mid X \times\{0\}=f$ and $p \tilde{h}=h$.

Theorem (2.5) suggests the following definition.

Definition (2.6). If $\mathcal{F}=(E, p, B), \mathcal{F}^{\prime}=\left(E^{\prime}, p^{\prime}, B\right)$ are (Hurewicz) fiber spaces over the same base $B$, then, $\mathscr{F}^{\prime}$ is called a fiber subspace of $\mathcal{F}$ provided $E^{\prime} \subset E, p^{\prime}=p \mid E^{\prime}$ and $\mathcal{F}$ admits a lifting function $\Lambda$ with the additional property that $\Lambda\left(e^{\prime}, \omega\right)$ is a path in $E^{\prime}$ whenever $e^{\prime} \in E^{\prime}$.

Thus, Theorem (2.5), states that if $\mathcal{F}^{\prime}$ is a locally trivial fiber subspace of $\mathcal{F}$ with common paracompact base $B$, then $\mathcal{F}^{\prime}$ is a fiber subspace of $\mathcal{F}$.

Theorem (2.6). If $\mathcal{F}=(E, p, B)$ and $\mathcal{F}^{\prime}=\left(E^{\prime}, p^{\prime}, B\right)$ are fiber spaces (Hurewicz) over the same base $B$ with $E^{\prime} \subset E$ and $p^{\prime}=p \mid E^{\prime}$, then $\mathcal{F}^{\prime}$ is a fiber subspace of $\mathcal{F}$ whenever $E$ is an ANR, $B$ is metric and $E^{\prime}$ is closed in $E$.

Proof. Let $\Omega_{p}=\{(e, \omega) \mid p(e)=\omega(0)\} \subset E \times B^{I}, \Omega_{p^{\prime}}=\left\{\left(e^{\prime}, \omega\right) \mid p^{\prime}\left(e^{\prime}\right)=\omega(0)\right\}$ $\subset E^{\prime} \times B^{I}$, and $\Lambda: \Omega_{p} \rightarrow E^{I}, \Lambda^{\prime}: \Omega_{p^{\prime}} \rightarrow E^{\prime I}$ (regular) lifting functions for $\mathcal{F}$ and $\mathcal{F}^{\prime}$, respectively. Furthermore, let $W=\left\{(e, \omega) \in \Omega_{p} \mid \omega=\right.$ constant path at $\left.p(e)\right\}$. Then, if $X=\Omega_{p} \times I, A=\left(\Omega_{p} \times\{0\}\right) \cup(W \times I) \cup\left(\Omega_{p}^{\prime} \times I\right),(X, A)$ is a metric pair with $A$ closed in $X$. Define $H: X \rightarrow E$ by $H(e, \omega, t)=\Lambda(e, \omega)(t)$ and $\mathrm{g}: A \rightarrow E$ by

$$
\begin{array}{ll}
g\left(e^{\prime}, \omega, t\right)=\Lambda^{\prime}\left(e^{\prime}, \omega\right)(t), & \text { if }\left(e^{\prime}, \omega\right) \in \Omega_{p^{\prime}}, 0 \leqq t \leqq 1, \\
g(e, \omega, t)=e, & \text { if }(e, \omega) \in W, 0 \leqq t \leqq 1, \\
g(e, \omega, 0)=e, & \text { if }(e, \omega) \in \Omega_{p} .
\end{array}
$$

Then, if $h=H \mid A$, we have by Proposition 1 of [1], $g \sim f h: A \rightarrow E$. Thus, by FHET (2.2), $g$ is extendable to a map $G: X \rightarrow E$, such that $G \sim_{f} H$. Hence, if we define

$$
\Lambda^{\prime \prime}(e, \omega)(t)=G(e, \omega, t),(e, \omega, t) \in \Omega_{p} \times I,
$$

$\Lambda^{\prime \prime}$ is the required lifting function and $\mathcal{F}^{\prime}$ is a fiber subspace of $F$.

REMARK. Thus, Corollary (2.6), which is valid for $\mathcal{F}^{\prime}$ a fiber subspace of $\mathcal{F}$, is valid when $\mathcal{F}^{\prime}, \mathcal{F}$ are locally trivial fiber spaces over the same metric space $B$, provided $E^{\prime} C E$ and $p^{\prime}=p \mid E$ and if further $E^{\prime}$ is a closed subset of the ANR $E$. There need not be in this situation, therefore, any connection between the local product structures of $\mathcal{F}$ and $\mathcal{F}^{\prime}$, respectively.

It may be of interest to mention that the existence of a lifting function which applies simultaneously to $\mathscr{F}$ and $\mathcal{F}^{\prime}$ as in Theorem (2.6) provides simpler proofs in many situations. For example:

TheOREM (2.7). Let $\mathcal{F}^{\prime}=\left(E^{\prime}, p^{\prime}, B\right)$ denote a fiber subspace of $\mathcal{F}=(E, p, B)$, with respective fibers $F^{\prime}$ and $F$. Then, there is a spectral sequence associated with a filtration of the singular $G$-chains, $C_{*}\left(E, E^{\prime}, G\right)$ whose $E_{2}$-term is naturally isomorphic to $H\left(B ; H\left(F, F^{\prime}, G\right)\right.$ ) (local coefficients). 
Proof. Essentially word-for-word the argument given for the Leray-Serre Theorem in [3] or [6].

Corollary (2.8) (Тном). Let $\mathcal{F}=\left(E, p, B, F=R^{n}\right)$, denote an n-plane bundle over a paracompact base $B$ and let $E^{\prime}=E-(0$-section $), F^{\prime}=F-\{0\}$, $p^{\prime}=p \mid E^{\prime}$, then $\mathcal{F}^{\prime}=\left(E^{\prime}, p^{\prime}, B, F^{\prime}\right)$ is a fiber subspace of $\mathcal{F}$. Hence, there is a natural isomorphism

$$
H_{n+k}\left(E, E^{\prime} ; G\right) \approx H_{k}\left(B, H_{n}\left(F, F^{\prime} ; G\right)\right), \quad k \geqq-n
$$

where local coefficients appear on the right. Hence, if $\pi_{1}(B)$ operates trivially on $H\left(F, F^{\prime} ; G\right)$, we have

$$
H_{n+k}\left(E, E^{\prime} ; G\right) \approx H_{k}(B ; G), \quad k \geqq-n
$$

e.g. if $G=Z$ and we have an oriented bundle or if $G=Z_{2}$.

REMARK. Theorem (2.7) is, of course, valid when $\mathcal{F}$ and $\mathcal{F}^{\prime}$ are fiber spaces in the sense of Serre [8], provided the base $B$ is common to both $\mathcal{F}$ and $\mathcal{F}^{\prime}$, $E^{\prime} \subset E$ and $p^{\prime}=p \mid E^{\prime}$. The proof in this case is more cumbersome.

3. Applications. As an immediate consequence of the FHET and its proof we have the classical result:

Corollary (3.1). Let $\mathcal{F}=(E, p, B)$ be a fiber space and assume that $E$ is an ANR. Let $A$ be a closed subset of $B$. Suppose $\sigma, \sigma^{\prime}: A \rightarrow E$ are partial cross sections which are homotopic in the sense of $[9$, p. 167]. Then if $\sigma$ is extendable to a full cross section, $\sigma^{\prime}$ is also extendable.

REMARK. The same result holds if $(B, A)$ is an ANR pair.

The following Corollary is also immediate.

Corollary (3.2). Let $\mathcal{F}=(E, p, B)$ be a fiber space and let $(X, A)$ be an arbitrary pair of spaces. If $E$ has the homotopy extension property with respect to $(X, A)$ so does the fiber $F_{b}$, for every $b \in B$.

Let $b_{0}$ be a base point in $B$ and let $\Omega(B)$ be the space of loops based at $b_{0}$. If $\mathcal{F}=(E, p, B)$ is a fiber space over $B$, any lifting function $\Lambda$ induces a map $\phi: \Omega(B) \times F_{b_{0}} \rightarrow F_{b_{0}}$ given by

$$
\phi(\beta, x)=\Lambda(x, \beta)(1), \quad \beta \in \Omega(B), x \in F_{b_{0}} .
$$

$\phi$ is called the loop action induced by $\Lambda$ and is uniquely determined up to a homotopy class. Since $\Lambda$ is regular, then $\phi\left(\tilde{b}_{0}, x\right)=x$, where $\tilde{b}_{0}$ denotes the constant loop at $b_{0}$. Our next theorem has to do with the realization of loop actions via lifting functions.

TheOREM (3.3). Let $\mathcal{F}=(E, p, B)$ be a fiber space over a metric base $B$, with fiber $F=p^{-1}\left(b_{0}\right)$, and suppose that $\phi$ is a loop action induced by a lifting function $\Lambda$. Let $\phi^{\prime}: \Omega(B) \times F \rightarrow F$ be a map which is homotopic to $\phi$ and such that $\phi^{\prime}\left(\bar{b}_{0}, x\right)=x, x \in F$. Then, if $E$ is an ANR there is a (regular) lifting function $\Lambda^{\prime}$ for $\mathcal{F}$ which induces $\phi^{\prime}$. 
Proof. By hypothesis there is a homotopy $H: \Omega(B) \times F \times I \rightarrow F$ such that $H_{0}=\phi, H_{1}=\phi^{\prime}$.

We first show that we can assume $H\left(\tilde{b}_{0}, x, t\right)=x, 0 \leqq t \leqq 1, x \in F$. Let

$$
A=(\Omega(B) \times F \times\{0\}) \cup(\Omega(B) \times F \times\{1\}) \cup\left(\tilde{b}_{0} \times F \times I\right)
$$

and define a homotopy $G: A \times I \rightarrow F$ as follows.

$$
\begin{aligned}
G(\beta, x, 0, s) & =H(\beta, x, 0)=\phi(\beta, x), \\
G\left(\tilde{b}_{0}, x, t, s\right) & =H\left(\tilde{b}_{0}, x,(1-s) t\right), \\
G(\beta, x, 1, s) & =H\left(\beta, H\left(\tilde{b}_{0}, x, 1-s\right), 1\right) \\
& =\phi^{\prime}\left(\beta, H\left(\tilde{b}_{0}, x, 1-s\right)\right),
\end{aligned}
$$

$\beta \in \Omega(B), x \in F, 0 \leqq s, t \leqq 1$. Note that $G_{1}\left(\tilde{b}_{0}, x, t\right)=x$ and that $G_{0}$ has an extension to all of $\Omega(B) \times F \times I$, namely $H$. By Corollary (3.2), $F$ has the homotopy extension property with respect to metric pairs, and therefore, $G_{1}$ can be extended to all of $\Omega(B) \times F \times I$, giving a new homotopy with the required property.

Now, let $\Omega_{p}^{*}=\left\{(\omega, e) \mid(e, \omega) \in \Omega_{p}\right\}$, let $\tilde{B}$ be the set of constant paths in $B$ and let $K=(\Omega(B) \times F) \cup(\widetilde{B} \times E) \cap \Omega_{p}^{*}$. $K$ is a closed subset of $\Omega_{p}^{*}$. Define a homotopy $H^{\prime}: K \times I \rightarrow E$ as follows.

$$
\begin{aligned}
& H^{\prime}(\beta, x, t)=H(\beta, x, t), \quad \beta \in \Omega(B), x \in F, \\
& H^{\prime}(\tilde{b}, e, t)=e, \quad e \in E, p(e)=b .
\end{aligned}
$$

$H^{\prime}$ is well-defined and continuous and the map $\bar{H}_{0}: \Omega_{p}^{*} \rightarrow E$ given by

$$
\bar{H}_{0}(\omega, e)=\Lambda(e, \omega)(1)
$$

is an extension of $H_{0}^{\prime}$. By the FHET, $H_{1}^{\prime}$ is extendable to all of $\Omega_{p}^{*}$ giving a map $\bar{H}_{1}: \Omega_{p}^{*} \rightarrow E$ such that:

$$
\begin{aligned}
\bar{H}_{1} \mid \Omega(B) \times F & =\phi^{\prime}, \\
\bar{H}_{1}(\tilde{b}, e) & =e, \quad p(e)=b, \\
p \bar{H}_{1}(\omega, e) & =\omega(1) .
\end{aligned}
$$

If $\omega \in B^{I}$, for every $0 \leqq s \leqq 1$ define a new path $\omega_{8} \in B^{I}$ by $\omega_{8}(t)=\omega(s t), 0 \leqq t \leqq 1$.

Finally, the required (regular) lifting function $\Lambda^{\prime}$ which induces $\phi^{\prime}$ is given by

$$
\Lambda^{\prime}(e, \omega)(s)=\bar{H}_{1}\left(\omega_{s}, e\right), \quad 0 \leqq s \leqq 1,(e, \omega) \in \Omega_{p} .
$$

4. Further results. In $[1$, p. 7$]$ the following question was raised:

Question. If $(E, p, B)$ is a fiber space in which $E$ is separable metric and $B$ as well as all the fibers are separable metric ANR's, is $E$ an ANR?

In this section we will show that the answer is affirmative if $E$ is finite dimensional (Theorem 4.13), and more generally will consider the following problem. Let $(E, p, B)$ be a fiber space. If $E$ is an ANR what can be said 
about $B$ and the fibers? Conversely, if $B$, and one or all the fibers are ANR's, what conclusions can be shown about $E$ ?

Definition (4.1). A space $B$ is weakly locally contractible (w.l.c.), if for every $b \in B$, there is an open set $U$, containing $b$ which is contractible to $b$ in $B$.

Weak local contractibility is a homotopy type invariant, and in fact a dominance invariant, $[1$, p. 1$]$. Hence, if $(E, p, B)$ is a fiber space over a 0 connected base, then if one fiber is w.l.c., every fiber is w.l.c. We also have the following lemma whose proof is left to the reader.

Lemma (4.2). Let (E, $p, B)$ be a fiber space. Suppose $B$ is 0-connected and w.l.c. Then, if $F_{b_{0}}$ is w.l.c. for some $b_{0} \in B, E$ is w.l.c.

It might be pointed out that it is possible for $E$ and all the fibers to be w.l.c. without $B$ being w.l.c., and for $E$ and $B$ to be w.l.c. without any of the fibers being w.l.c. ( $\$ 5$ and $\S 4$ of $[1])$.

Theorem (4.3). Let $(E, p, B)$ be a fiber space in which $E$ is an ANR and $B$ is 0-connected. Then if $F_{b_{0}}$ is w.l.c. for some $b_{0} \in B, F_{b}$ is an ANR for every $b \in B$.

Proof. First, let $X$ be any separable metric space which is w.l.c. and which has the homotopy extension property with respect to metric pairs. Let $(Z, A)$ be such a pair. For any $x_{0} \in X$, let $U$ be an open set containing $x_{0}$, which is contractible in $X$ to $x_{0}$; and let $f: A \rightarrow U$ be a given map. If $f$ is considered as a map into $X$ it is homotopically trivial, and can be extended to a map $Z \rightarrow X$. $F^{-1}(U)$ is an open set of $Z$ containing $A$ and $F \mid F^{-1}(U)$ is an extension of $f$ with values in $U$. Hence $X$ is a local ANR and consequently an ANR by a theorem of Hanner [3].

Now, by Corollary (3.2), each fiber $F_{b}$ has the homotopy extension property with respect to metric pairs and since each $F_{b}$ is w.l.c. the result follows.

The lemma is false without the w.l.c. condition on $F_{b_{0}}$ as will be seen in the next section.

We introduce now some notation. Let $X$ and $Y$ be topological spaces and $f: X \rightarrow Y$ a given map. To the triple $(X, f, Y)$ we associate a fiber space defined in the following way.

Let $\Omega_{f} \subset X \times Y^{I}$ be given by

$$
\Omega_{f}=\{(x, \omega) \mid \omega(0)=f(x)\} .
$$

$\Omega_{f}$ is given the relative topology. If we set $p_{f}(x, \omega)=\omega(1)$ it is easily seen that the triple $\left(\Omega_{f}, p_{f}, Y\right)$ is a fiber space. If $y_{0}$ is a base point in $Y$, let $A_{f}=p_{f}^{-1}\left(y_{0}\right)$, and let $\pi(x, \omega)=x,(x, \omega) \in A_{f}$. The triple $\left(A_{f}, \pi, X\right)$ is also a fiber space and $\pi^{-1}\left(x_{0}\right)=\Omega\left(Y, y_{0}\right)$, if $y_{0}=f\left(x_{0}\right)$. If we had started with a fiber space $(E, p, B)$, then as is well known, $E$ and $\Omega_{p}$ are fiber homotopy equivalent; and in particular, choosing a base point $b_{0}$ in $B, p^{-1}\left(b_{0}\right)=F$ and $A_{p}$ are of the same homotopy type. 
Also, if $\left(E, F, x_{0}\right)$ is an arbitrary pair with base point, let $\Gamma\left(E, F, x_{0}\right)$ be the subset of $E^{I}$ defined by

$$
\Gamma\left(E, F, x_{0}\right)=\left\{\omega: I \rightarrow E \mid \omega(0)=x_{0}, \omega(1) \in F\right\} .
$$

This subset is to be given the relative topology.

Our first objective is to prove that if $E$ and $F$ are ANR's $\Gamma\left(E, F, x_{0}\right)$ is an ANR, and similarly, that if $X$ and $Y$ are ANR's $\Omega_{f}$ is an ANR. We will need the following simple lemma.

Lemma (4.4). Let $Z$ be an ANR and $(X, A)$ a metric pair. Let $f: X \rightarrow Z^{I}$ and $g: A \rightarrow Z^{I}$ be maps such that $f \sim g$ on $A$, keeping end points fixed, that is, there is $a$ homotopy $H: A \times I \rightarrow Z^{I}$ such that $H_{0}=f \mid A, H_{1}=g, H(x, s)(0)=f(x)(0)$ $=g(x)(0), H(x, s)(1)=f(x)(1)=g(x)(1)$. Then $g$ has an extension $g^{\prime}$ to all of $X$ such that $g^{\prime}(x)(0)=f(x)(0), g^{\prime}(x)(1)=f(x)(1)$.

Proof. The triple $\left(Z^{I}, \gamma \times \gamma, Z \times Z\right)$, where $\gamma \times \gamma(\omega)=(\omega(0), \omega(1))$ is a fiber space. By hypothesis $f \sim_{f} g$ on $A$ and the FHET implies the existence of $g^{\prime}$.

Lemma (4.5). If (E, F) is an ANR pair, then $\Gamma\left(E, F, x_{0}\right)$ is an ANR.

Lemma (4.6). If $(X, f, Y)$ is a triple such that $X$ and $Y$ are ANR's then $\Omega_{f}$ is an ANR.

We will prove Lemma (4.5), the proof of Lemma (4.6) being essentially the same except for some obvious modifications.

Proof of Lemma (4.5). By Hanner [4] it is sufficient to prove that $\Gamma\left(E, F, x_{0}\right)$ is a local ANR. Let $\omega_{0}$ be any point in $\Gamma\left(E, F, x_{0}\right)$. Since $E$ is an ANR, it is U.L.C. [8], therefore there exists a neighborhood $U$ of $\omega_{0}(1)$ and a map $\phi: U \times U \rightarrow E^{I}$ such that:

$$
\begin{array}{ll}
\phi(x, y)(0)=x, & (x, y) \in U \times U, \\
\phi(x, y)(1)=y, & (x, y) \in U \times U, \\
\phi(x, x)(s)=x, & 0 \leqq s \leqq 1, x \in U .
\end{array}
$$

If we let $M=\left\{\omega: I \rightarrow E \mid \omega(0)=x_{0}, \omega(1) \in U\right\}$, then $M$ is an ANR since it is an open subset of $P\left(E, x_{0}\right)$. Hence,

$$
K=M \cap \Gamma\left(E, F, x_{0}\right)=\left\{\omega: I \rightarrow E \mid \omega(0)=x_{0}, \omega(1) \in F \cap U\right\}
$$

is an open subset of $\Gamma\left(E, F, x_{0}\right)$ and $\omega_{0} \in K$. We will show that $K$ is an ANR.

Let $(Z, A)$ be a metric pair, and $f: A \rightarrow K$ a given map. $F \cap U$ is an open subset of $F$ and hence an ANR. It follows that considering $f$ as a map $A \rightarrow M$ there is an open set $V \supseteq A$ and two maps

$$
\begin{array}{ll}
h: V \rightarrow M, & h \mid A=f, \\
g: V \rightarrow F \cap U, & g \mid A=f(z)(1), z \in A .
\end{array}
$$

Define a map $\gamma: V \rightarrow K$ by 


$$
\gamma(z)=h(z) \circ \phi[h(z)(1), g(z)], \quad z \in V
$$

where $\circ$ denotes the usual path multiplication. Then if $z \in A$

$$
\gamma(z)=f(z) \circ \phi[f(z)(1), f(z)(1)]
$$

so that:

$$
\gamma(z)(t)=\left\{\begin{array}{lr}
f(z)(2 t), & 0 \leqq t \leqq 1 / 2, \\
f(z)(1), & 1 / 2 \leqq t \leqq 1
\end{array}\right.
$$

$\gamma$ is then homotopic to $f$ keeping end points fixed. By Lemma (4.4) there exists a map $f^{\prime}: V \rightarrow E^{I}$ such that $f^{\prime} \mid A=f$,

$$
f^{\prime}(z)(0)=\gamma(z)(0)=x_{0}, \quad f^{\prime}(z)(1)=\gamma(z)(1) \in F \cap U .
$$

$f^{\prime}$ is a map $V \rightarrow K$ and it follows that $K$ is an ANR.

TheOREM (4.7). Let $(E, p, B)$ be a fiber space such that $E$ is an ANR, $B$ is 0 -connected and $F_{b_{0}}$ is w.l.c. for some $b_{0} \in B$. Then for every $b \in B, F_{b}$ and $\Omega(B, b)$ are ANR's.

Proof. By Theorem (4.3), $F_{b}$ is an ANR for every $b \in B$, and by Lemma (4.5) $\Gamma\left(E, F_{b}, x_{0}\right)$ is an ANR for any $x_{0} \in F_{b}$. Let $\Lambda$ be a lifting function and define a map $\mu: \Omega(B, b) \rightarrow \Gamma\left(E, F_{b}, x_{0}\right)$ by

$$
\mu(\beta)=\Lambda\left(x_{0}, \beta\right), \quad \beta \in \Omega(B, b) .
$$

Now, $p$ induces a map $\bar{p}: \Gamma\left(E, F, x_{0}\right) \rightarrow \Omega(B, b)$ given by

$$
\bar{p}(\alpha)(t)=p(\alpha(t)), \quad \alpha \in \Gamma\left(E, F, x_{0}\right),
$$

and $p \mu=1$. It follows that $\Omega(B, b)$ is an ANR since it is imbedded in $\Gamma\left(E, F, x_{0}\right)$ as a retract.

Theorem (4.7) has a converse, in the following sense:

TheOREM (4.8). Let $(E, p, B)$ be a fiber space such that $E$ is an ANR and $\Omega\left(B, b_{0}\right)$ is w.l.c. for some $b_{0} \in B$. Then if $E$ is 0 -connected, $F_{b}$ and $\Omega(B, b)$ are ANR's for every $b \in B$.

Proof. Recall the notation introduced after Theorem (4.3) and consider the fiber space $\left(A_{p}, \pi, E\right)$ associated with the map $p: E \rightarrow B$. If $x_{0} \in F_{b_{0}}$, then $\pi^{-1}\left(x_{0}\right)=\Omega\left(B, b_{0}\right)$. Lemma (4.2) tells us that $A_{p}$ is w.l.c. But since $F_{b_{0}}$ and $A_{p}$ have the same homotopy type, $F_{b_{0}}$ is w.l.c. and Theorem (4.7) applies to complete the argument.

Theorem (4.9). Let $(E, p, B)$ be a fiber space such that for some $b_{0} \in B$, $\Omega\left(B, b_{0}\right)$ and $F_{b_{0}}$ are dominated by ANR's. Then $\Omega\left(E, x_{0}\right)$ is dominated by an ANR, for any $x_{0} \in F_{b_{0}}$.

We will need the following lemmas. 
LEMMA (4.10). Let $f: X \rightarrow Y$ denote a map and suppose $\phi: X \rightleftarrows Z: \psi$ are maps such that $\psi \phi \sim 1$ (i.e., $Z$ dominates $X)$. Then, if $g=f \psi$, there is induced a fiberwise dominance

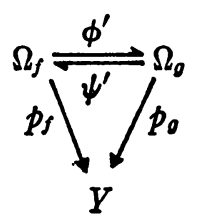

i.e., $\psi^{\prime} \phi^{\prime} \sim{ }_{f} 1$.

Proof. Since $\psi \phi \sim 1$, there is a map $k: X \rightarrow X^{I}$ such that $k(x)(0)=\psi \phi(x)$ and $k(x)(1)=x$. Define

and

$$
\phi^{\prime}(x, \omega)=(\phi(x), f(k(x)) \circ \omega), \quad(x, \omega) \in \Omega_{f}
$$

$$
\psi^{\prime}(z, \omega)=(\psi(z), \omega), \quad(z, \omega) \in \Omega_{g} .
$$

It is obvious that $\psi^{\prime} \phi^{\prime} \sim_{f} 1$.

Lemma (4.11). Let $f: X \rightarrow Y$ denote a map and suppose $\alpha: Y \rightleftarrows W: \beta$ are maps such that $\beta \alpha \sim 1$ (i.e., $W$ dominates $Y$ ). Then, if $h=\alpha f, A_{f}$ based at $y_{0}$ is dominated by $A_{h}$ based at $w_{0}=\alpha\left(y_{0}\right)$.

Proof. The proof here is similar to (4.10) and is omitted.

Lemma (4.12). Let $f: X \rightarrow Y$ denote a map. If $X$ and $Y$ are dominated by ANR's, $A_{f}$ is dominated by an ANR.

Proof. Consider the diagram

$$
\begin{aligned}
& X \underset{\psi}{\stackrel{\phi}{\rightleftarrows}} Z \\
& f \downarrow \\
& Y \underset{\beta}{\stackrel{\alpha}{\rightleftarrows}} W
\end{aligned}
$$

where $\psi \phi \sim 1, \beta \alpha \sim 1$ and $Z$ and $W$ are ANR's. Applying the previous lemmas $A_{\alpha f \psi}$ dominates $A_{\alpha f}$ (based at $w_{0}=\alpha\left(y_{0}\right)$ ), and $A_{\alpha f}$ dominates $A_{f}$ (based at $y_{0}$ ). But, since $\Omega_{\alpha f \psi}$ is an ANR (Lemma (4.6)) fibered over the ANR $W$, we may conclude that the fiber here, which is $A_{\alpha f \omega}$, is also an ANR by [1, p. 7].

Proof of Theorem (4.9). Let $\Lambda$ denote a lifting function for the fiber space $(E, p, B)$ and define a map $f: \Omega\left(B, b_{0}\right) \rightarrow F_{b_{0}}$ by

$$
f(\omega)=\Lambda\left(x_{0}, \omega\right)(1), \quad \omega \in \Omega\left(B, b_{0}\right) .
$$

Consider the fiber space $\left(\Omega_{f}, p_{f}, F_{b_{0}}\right)$ associated with this map and corresponding fiber $A_{f}$ based at $x_{0} \in F_{b_{0}}$. By Lemma (4.12) $A_{f}$ is dominated by an ANR and hence we only have to show that $A_{f}$ dominates $\Omega\left(E, x_{0}\right)$. As shown in Proposition 1 of [1], there is a homotopy $H: E^{I} \times I \rightarrow E^{I}$ with the properties 
(i) $\quad H_{0}(\alpha)=\Lambda(\alpha(0), p(\alpha)), \quad H_{1}(\alpha)=\alpha, \alpha \in E^{I}$

(ii) $p(H(\alpha, s))=p(\alpha), \quad 0 \leqq s \leqq 1, \quad \alpha \in E^{I}$.

Define $h: \Omega\left(E, x_{0}\right) \rightarrow F_{b_{0}}^{I}$ by

$$
h(\omega)(s)=H(\omega, s)(1), \quad 0 \leqq s \leqq 1, \omega \in \Omega\left(E, x_{0}\right) .
$$

Then $h(\omega)$ is a path which starts at $f(p \omega)$ and ends at $x_{0}$. Note here that the map $\bar{h}: \Omega\left(E, x_{0}\right) \rightarrow \Omega\left(E, x_{0}\right)$ given by

$$
\bar{h}(\omega)=\Lambda\left(x_{0}, p(\omega)\right) \circ h(\omega)
$$

is homotopic to the identity. Now, define $\mu: A_{f} \rightarrow \Omega\left(E, x_{0}\right)$ by

$$
\mu(\beta, \alpha)=\Lambda\left(x_{0}, \beta\right) \circ \alpha, \quad(\beta, \alpha) \in A_{f}
$$

and $\gamma: \Omega\left(E, x_{0}\right) \rightarrow A_{f}$ by

$$
\gamma(\omega)=(p(\omega), h(\omega)), \quad \omega \in \Omega\left(E, x_{0}\right) .
$$

Then,

$$
\mu \gamma(\omega)=\Lambda\left(x_{0}, p(\omega)\right) \circ h(\omega)=\bar{h}(\omega)
$$

and hence $\mu \gamma \sim 1$ and $A_{f}$ dominates $\Omega\left(E, x_{0}\right)$.

REMARK. Theorem (4.9) cannot be strengthened by assuming that $F_{b_{0}}$ and $\Omega\left(B, b_{0}\right)$ are ANR's and concluding that $\Omega\left(E, x_{0}\right)$ is an ANR. That this is false will be shown by the first example of the next section.

THEOREM (4.13). Let $(E, p, B)$ be a fiber space in which $B$ and all the fibers are ANR's. Then $E$ is locally contractible. Hence, if in addition $E$ is separable metric and finite dimensional, $E$ is an ANR.

Proof. Let $x_{0}$ be a point of $E$ and $F_{b_{0}}$ the fiber containing $x_{0}$. Let $U_{1}$ be an open subset of $E$ containing $x_{0} . V_{1}=U_{1} \cap F_{b_{0}}$ is open in $F_{b_{0}}$, and by the local contractibility of $F_{b_{0}}$ there is an open set $V_{2} \subset F_{b_{0}}$, such that $x_{0} \in V_{2} \subset V_{1}$, and a map $\psi: V_{2} \times I \rightarrow V_{1}$ such that

$$
\begin{array}{ll}
\psi(x, 0)=x, & x \in V_{2}, \\
\psi(x, 1)=x_{0}, & x \in V_{2}, \\
\psi\left(x_{0}, s\right)=x_{0}, & 0 \leqq s \leqq 1 .
\end{array}
$$

There is also an open subset $U_{2}$ of $E$ such that $x_{0} \in U_{2}, U_{2} \cap F_{b_{0}}=V_{2}$. We can assume that $p\left(U_{1}\right)=M$ is contractible in $B$ to $b_{0}$ keeping $b_{0}$ fixed, so that there is a map $\gamma: M \rightarrow B^{I}$ with the properties

$$
\begin{aligned}
\gamma(b)(0) & =b, & & b \in M, \\
\gamma(b)(1) & =b_{0}, & & b \in M, \\
\gamma\left(b_{0}\right)(s) & =b_{0}, & & 0 \leqq s \leqq 1 .
\end{aligned}
$$


Now, if $\Lambda$ is a (regular) lifting function for $(E, p, B), \Lambda$ induces a map $\mu: U_{\mathbf{1}}$ $\rightarrow E^{I}$ given by

$$
\mu(x)=\Lambda(x, \gamma(p(x))), \quad x \in U_{1} .
$$

In particular, $\mu\left(x_{0}\right)$ is the constant path at $x_{0}$. Let $L=\left\{\omega: I \rightarrow E \mid \omega(I) \subset U_{2}\right\}$. $L$ is an open subset of $E^{I}$ and $\mu\left(x_{0}\right) \in L$. Since $\mu$ is continuous, there is an open set $U_{3}$ of $E$ containing $x_{0}$ such that $\mu\left(U_{3}\right) \subset L$ and $U_{3} \subset U_{1}$. If $x \in U_{3}$, then

$$
\begin{aligned}
& \mu(x)(0)=x, \\
& \mu(x)(1) \in U_{2} \cap F_{b_{0}}=V_{3} \subset V_{2}, \\
& \mu(x)(t) \in U_{2} \subset U_{1}, \quad 0 \leqq t \leqq 1 .
\end{aligned}
$$

We can now define a contraction $\mu^{\prime}: U_{3} \times I \rightarrow U_{1}$ keeping $x_{0}$ fixed by:

$$
\mu^{\prime}(x)(t)=\left\{\begin{array}{l}
\mu(x)(2 t), \quad 0 \leqq t \leqq 1 / 2 \\
\psi(\mu(x)(1), 2 t-1), \quad 1 / 2 \leqq t \leqq 1 .
\end{array}\right.
$$

Thus $E$ is locally contractible at $x_{0}$. The second part of the theorem follows from the classical result that a separable metric space which is finite dimensional and locally contractible is an ANR.

5. Two examples.

(5.1). A fiber space over the interval $[0,1]$ in which only one fiber is an ANR.

Let $X$ be a subset of Euclidean $n$-space $E^{n}$. The cone over $X, C(X)$, is the subset of $E^{n+1}$ defined by

$$
C(X)=(t, t x), \quad 0 \leqq t \leqq 1, x \in X .
$$

Let $C^{2}(X)=C[C(X)]$, i.e., $C^{2}(X)$ is the subset of $E^{n+2}$ given by

$$
C^{2}(X)=(s, s t, s t x), \quad 0 \leqq t \leqq 1,0 \leqq s \leqq 1, x \in X .
$$

Let $\pi: C^{2}(X) \rightarrow I$ be the projection

$$
\pi(s, s t, s t x)=s .
$$

Theorem (5.2). $\left(C^{2}(X), \pi, I\right)$ is a fiber space.

Proof. Suppose $(E, p, B)$ is a fiber space and $X$ is a subset of $E$ which is a fiber retract of $E$, that is, there is a map $\Phi: E \rightarrow X$ such that $\Phi(x)=x, x \in X$; $p \Phi(x)=p(x), x \in E$. Then, $(X, p, B)$ is a fiber space. To see this, let $(x, \omega)$ be a pair such that $p(x)=\omega(0), x \in X, \omega \in B^{I}$, and set

$$
\Lambda^{\prime}(x, \omega)(t)=\Phi(\Lambda(x, \omega)(t)), \quad 0 \leqq t \leqq 1,
$$

where $\Lambda$ is a lifting function for $(E, p, B) . \Lambda^{\prime}$ is a lifting function for $(X, p, B)$.

Now $C(X) \times I=\{(s, t, t x) \mid x \in X, 0 \leqq t \leqq 1,0 \leqq s \leqq 1\}$ is a fiber space over $I$ using the projection $\pi(s, t, t x)=s$. Clearly $C^{2}(X) \subset C(X) \times I$. Define a fiber retraction $\Phi: C(X) \times I \rightarrow C^{2}(X)$ as follows: 


$$
\Phi(s, t, t x)= \begin{cases}(s, s, s x) & \text { if } t \geqq s, \\ (s, t, t x) & \text { if } t \leqq s .\end{cases}
$$

$\Phi$ is well defined and continuous, and if $z \in C^{2}(X), z=\left(s^{\prime}, s^{\prime} t^{\prime}, s^{\prime} t^{\prime} x\right)=(s, t, t x)$ where $t=t^{\prime} s^{\prime}, s^{\prime}=s$, so that $\Phi(z)=z$. $\left(C^{2}(X), \pi, I\right)$ is then a fiber space such that $\pi^{-1}(s)=C(X), s \neq 0, \pi^{-1}(0)=$ a single point.

If, for instance, we let $X=\{0\}+\left\{1 / 2^{n}\right\}, n=1,2, \cdots$, i.e., a convergent sequence of reals, $C(X)$ is not an ANR and we have the required example. $C^{2}(X)$ is not locally contractible, showing that the requirement in Theorem (4.13) that all the fibers be ANR's, is crucial. $\Omega\left[C^{2}(X), v\right]$, where $v$ is the vertex of the double cone, is not an ANR, so that, as remarked after the proof of Theorem (4.9), it is possible to have a fiber space such that $\Omega\left(B, b_{0}\right)$ and $F_{b_{0}}$ are ANR's, but $\Omega\left(E, x_{0}\right)$ is not an ANR, where $x_{0} \in F_{b_{0}}$.

(5.3). A fiber space in which the total space is an ANR but none of the fibers are ANR's.

Let $X_{n}=\prod_{i=1}^{\infty} S_{i}^{n}$ be a countable cartesian product of $n$-spheres. Choose a base point $x_{0}$ in $X_{n}$ and let $E_{n}=P\left(X_{n}, x_{0}\right)$ be the space of paths in $X_{n}$ which start at $x_{0}$. The triple $\left(E_{n}, \gamma, X_{n}\right)$, where $\gamma(\omega)=\omega(1), \omega \in E_{n}$, is a fiber space. It is clear that $\Omega\left(X_{n}\right)=\gamma^{-1}\left(x_{0}\right)$ is not w.l.c. since any neighborhood of $x_{0}$ contains a countable product of $n$-spheres. Hence, $\Omega\left(X_{n}\right)$ is not an ANR, and neither is $\gamma^{-1}(x)$ for any $x \in X_{n}$. On the other hand, $E_{n}$ is an ANR, in fact an AR. To see this we need only observe that $X_{n}$ has the homotopy extension property with respect to metric pairs since $S^{n}$ does, and this property is a product invariant. This implies that if $(Z, A)$ is a metric pair, and $f: A \rightarrow E_{n}$ is a given map, it can be extended to all of $Z$. Therefore, $E_{n}$ is an AR.

This shows that Theorem (4.3) and Theorem (4.7) are false without the w.l.c. condition on $F_{b_{0}}$.

\section{BIBLIOGRAPHY}

1. E. Fadell, On fiber spaces, Trans. Amer. Math. Soc. 90 (1959), 1-14.

2. - On fiber homotopy equivalence, Duke Math. J. 26 (1959), 699-706.

3. E. Fadell and W. Hurewicz, On the structure of higher differential operators in spectral sequences, Ann. of Math. (2)68 (1958), 314-347.

4. O. Hanner, Some theorems on ANR's, Ark. Mat. 1 (1951), 389-408.

5. W. Hurewicz, On the concept of fiber space, Proc. Nat. Acad. Sci. U.S.A. 41 (1955), 956-961.

6. W. Hurewicz and E. Fadell, On the structure of higher terms of the spectral sequence of a fiber space, Symposium International de Topologia Algebraica (Mexico City, 1958).

7. A. T. Lundell, Obstruction theory of principal fibre bundles, Trans. Amer. Math. Soc. 97 (1960), 161-192.

8. J. P. Serre, Homologie singuliere des espaces fibres, Ann. of Math. (2)54 (1951), 425-505.

9. N. Steenrod, The topology of fibre bundles, Princeton Univ. Press, Princeton, N. J., 1951.

UNIVERSITY OF Wisconsin, Madison, Wisconsin 